\title{
Differential Galois Theory and Lie Symmetries
}

\author{
David BLÁZQUEZ-SANZ ${ }^{\dagger}$, Juan J. MORALES-RUIZ ${ }^{\ddagger}$ and Jacques-Arthur WEIL $\S$ \\ $\dagger$ Universidad Nacional de Colombia, Colombia \\ E-mail: dblazquezs@unal.edu.co \\ URL: https://sites.google.com/a/unal.edu.co/dblazquezs/ \\ $\ddagger$ Universidad Politécnica de Madrid, Spain \\ E-mail: juan.morales-ruiz@upm.es \\ $\S$ Université de Limoges, France \\ E-mail: jacques-arthur.weil@unilim.fr
}

Received March 31, 2015, in final form November 11, 2015; Published online November 20, 2015

http://dx.doi.org/10.3842/SIGMA.2015.092

\begin{abstract}
We study the interplay between the differential Galois group and the Lie algebra of infinitesimal symmetries of systems of linear differential equations. We show that some symmetries can be seen as solutions of a hierarchy of linear differential systems. We show that the existence of rational symmetries constrains the differential Galois group in the system in a way that depends of the Maclaurin series of the symmetry along the zero solution.
\end{abstract}

Key words: linear differential system; Picard-Vessiot theory; differential Galois theory; infinitesimal symmetries

2010 Mathematics Subject Classification: 12H05; 34M15; 34A26

\section{Introduction}

Differential Galois theory and Lie symmetries are two different theoretical frameworks designed to deal with similar mathematical problems: the integration, reduction, classification and listing of solutions of differential equations. Both theories appeared simultaneously at the end of 19th century. However, the links between them remained hidden for a long time, mostly because of the apparent walls that separate mathematical disciplines. Differential Galois theory appears to be central to differential algebra. On the other hand, the theory of Lie symmetries belongs to the realm of local differential geometry. For a general exposition of both theories we refer the readers to $[8,21]$ and $[11,16]$ respectively. Throughout this paper, "differential Galois theory" refers to the Galois theory of systems of linear differential equations, also called the Picard-Vessiot theory.

The works of Ziglin, Morales, Ramis, Simó, Churchill and others show that differential Galois groups may measure obstructions to the existence of first integrals of Hamiltonian systems; regarding this approach to non-integrability of dynamical systems, see the recent survey [14] and references therein. This suggests an interplay between Lie symmetries and Galois group. Indeed, for Hamiltonian systems, the (musical) duality induced by the symplectic structure transforms a first integral into a Lie symmetry; so, obstructions to the existence of first integrals induce obstructions to the existence of Hamiltonian vector fields of Lie symmetries.

In [2], Ayoul and Zung study a more general definition of integrability (Bogoyavlensky integrability [6]), which includes directly Lie symmetries and they show again how the Galois groups of variational equations appear to give obstructions to the existence of such symmetries.

\footnotetext{
*This paper is a contribution to the Special Issue on Algebraic Methods in Dynamical Systems. The full collection is available at http://www.emis.de/journals/SIGMA/AMDS2014.html
} 
B. Malgrange has also suggested a link between Lie symmetries and his non-linear differential Galois theory (see, e.g., [12, Remark iii, p. 224]). The general idea is that differential Galois groups and Lie symmetries should be related in the sense that more symmetries should imply a smaller Galois group. Our results elaborate on this idea to turn it into precise statements which apply to systems of linear differential equations.

In order to link Lie symmetries with differential Galois theory, we follow a geometrical approach developed by the first two authors in [4]. Some general results about symmetries were already stated in [5, Section 6], but in a more general context of automorphic systems. In particular, it is implicit in [5] that the eigenring [3, 20] consists of vertical Lie symmetries. Our approach here is less abstract and more explicit (see Section 6.2). In connection with that, we point out that the relevance of the eigenring for the symmetries of linear differential equations was discovered independently by C. Jensen in the nice paper [9], but without any mention of a relationship with Picard-Vessiot theory.

The results contained in this work may be summarized as follows:

(a) The search for symmetries of systems of linear differential equations may be reduced to the search for a particular kind of symmetries, namely homogeneous polynomial vertical symmetries (Lemma 3.3, Proposition 3.5).

(b) We focus on polynomial vertical infinitesimal symmetries and show that these are solutions of associated linear differential equations that can be deduced from the given equation (via the Tannakian theory). The differential Galois theories of those equations is related to that of our original system (Theorem 6.2).

(c) The Galois group determines the Lie algebra of polynomial vertical symmetries with coefficients in the ground field (Theorem 6.4).

(d) Each non-trivial vertical polynomial symmetry with coefficients in the ground field places constrains on the Galois group. Thus, the bigger the symmetry algebra, the smaller the Galois group. In several cases, a single symmetry may force the group to be abelian or solvable (Theorems 6.10 and 6.13, Corollaries 6.1 and 6.11).

Section 2 contains the basic definitions of symmetries. Section 3 studies polynomial symmetries and establishes part (a) above. Section 4 gives a dictionary between graded polynomial vector fields, linear actions and corresponding linear differential systems. A geometric definition of the differential Galois group is given in Section 5 and the comparisons with symmetries are derived in Section 6.

The problem of linking differential Galois theory with Lie symmetries of differential operators has been studied by C. Athorne in [1] and by W.R. Oudshoorn and M. van der Put in [17]. This restriction to differential operators seems to complicate the relations between the Galois group and the Lie symmetries. So, in this work, we adopt a slightly different point of view. Instead of considering higher-order linear differential equations, we study systems of first-order linear differential equations. This leads us to a broader notion of infinitesimal symmetry which has an explicit relation with the differential Galois group. In Appendix A, we propose a comparison between the definitions of infinitesimal symmetries in the contexts of higher-order equations and first-order systems.

\section{Characteristic and vertical symmetries}

Let $\mathcal{U}$ be an open subset of the complex projective line $\mathbb{C P}_{1}$. By a function field $\mathcal{K}$ we mean a subfield of the field of meromorphic functions on $\mathcal{U}$ such that $\mathcal{K}$ contains the constants $\mathbb{C}$ and is closed with respect the derivation $\frac{d}{d x}$. Clearly, fields of rational functions, elliptic functions, etc. are function fields. As shown by Seidenberg in $[18,19]$, any differential field which is finitely 
generated over $\mathbb{Q}$ can be embedded into such a function field. We fix from now on the function field $\mathcal{K}$ and its domain $\mathcal{U}$.

We consider a system of linear differential equations with coefficients in the function field $\mathcal{K}$

$$
\frac{d y}{d x}=A(x) y, \quad y=\left(y_{1}, \ldots, y_{n}\right), \quad A(x) \in \mathfrak{g l}(n, \mathcal{K}) .
$$

The poles of $A(x)$ are called the singularities. The point at infinity may be considered as a singularity depending on its behavior after a suitable change of coordinates. The graphs of the solutions of (2.1) are the integral curves of the associated vector field:

$$
X=\frac{\partial}{\partial x}+\sum_{i, j=1}^{n} a_{i j}(x) y_{j} \frac{\partial}{\partial y_{i}},
$$

which is a meromorphic vector field in $\mathcal{U} \times \mathbb{C}^{n}$.

An infinitesimal symmetry of $X$ is an analytic vector field $Y$ defined in some open subset of $\mathcal{U} \times \mathbb{C}^{n}$ such that $[Y, X]$ and $X$ are linearly dependent in their common domain of definition. In particular, vector fields of the form $\gamma X$ where $\gamma$ is an analytic function defined in some open subset of $\mathcal{U} \times \mathbb{C}^{n}$ are infinitesimal symmetries of $X$. They are called characteristic symmetries of $X$. Since the definition of infinitesimal symmetries is local, we have sheaves of infinitesimal symmetries and characteristic symmetries of $X$ in $\mathcal{U} \times \mathbb{C}^{n}$.

The Lie bracket of two infinitesimal symmetries is also an infinitesimal symmetry. Hence, infinitesimal symmetries form a Lie algebra sheaf. Characteristic symmetries form an ideal of the Lie algebra sheaf of infinitesimal symmetries. An infinitesimal symmetry $Y$ is a vertical symmetry if it is tangent to the fibers of the canonical projection $\mathcal{U} \times \mathbb{C}^{n} \rightarrow \mathcal{U}$, that is $Y x=0$. Its expression in coordinates takes the form

$$
Y=\sum_{i=1}^{n} f_{i}(x, y) \frac{\partial}{\partial y_{i}} .
$$

If $Y$ is a vertical symmetry, then the Lie bracket $[X, Y]$ vanishes.

The Lie algebra sheaf of vertical symmetries is canonically isomorphic to the quotient Lie algebra sheaf of all infinitesimal symmetries modulo the ideal of characteristic symmetries. If $Y$ is an infinitesimal symmetry, we can take its vertical representative: $\tilde{Y}=Y-(Y x) X$.

By this reduction, the algebra of vertical symmetries is isomorphic to the algebra of infinitesimal symmetries modulo the ideal of characteristic symmetries. Thus, in order to study the symmetries of (2.1), it suffices to consider vertical symmetries. We consider symmetries that are defined in open subsets of the form $\mathcal{V} \times \mathbb{C}^{n}$ with $\mathcal{V} \subseteq \mathcal{U}$. Such symmetries can be seen as sections of a sheaf defined in $\mathcal{U}$.

Definition 2.1. The sheaf $\operatorname{sym}_{X}$ in $\mathcal{U}$ assigns to each open subset $\mathcal{V} \subseteq \mathcal{U}$ the Lie algebra of vertical infinitesimal symmetries of $\mathrm{X}$ defined in $\mathcal{V} \times \mathbb{C}^{n}$

$$
\operatorname{sym}_{X}(\mathcal{V})=\left\{Y \in \mathfrak{X}_{\text {an }}\left(\mathcal{V} \times \mathbb{C}^{n}\right) \mid[Y, X]=0 \text { and } Y x=0\right\},
$$

where $\mathfrak{X}_{\text {an }}\left(\mathcal{V} \times \mathbb{C}^{n}\right)$ stands for the Lie algebra of analytic vector fields in $\mathcal{V} \times \mathbb{C}^{n}$.

Our objective is to describe the sections of this sheaf $\mathbf{s y m}_{X}$ and its relation with the closed form solutions and the Picard-Vessiot theory of the system (2.1). From now on, when we mention a symmetry of $X$ we mean a section of $\mathbf{s y m}_{X}$, that is, a vertical infinitesimal symmetry.

Our definition of infinitesimal symmetries is considered, for instance, in the reference book [11], where the vertical symmetries are called shuffing symmetries. We prefer our terminology, because it has a clearer geometrical meaning in our context of linear differential equations (i.e., fiber bundles, although we will not use this geometrical terminology explicitly here). Actually, the relation $[X, Y]=0$ sometimes appears in the literature as being the direct definition of an infinitesimal symmetry of $X$, see for instance $[6,7]$. 


\section{Polynomial vertical symmetries}

\subsection{Polynomial vertical vector fields}

Definition 3.1. Let $Y$ be a vertical vector field defined in $\mathcal{V} \times \mathbb{C}^{n}$ with $\mathcal{V}$ an open subset of $\mathcal{U}$

$$
Y=\sum_{i=1}^{n} f_{i}(x, y) \frac{\partial}{\partial y_{i}} .
$$

We say that $Y$ is a polynomial vertical vector field when the components $f_{i}(x, y)$ are polynomials in the variables $y_{1}, \ldots, y_{n}$.

Example 3.2. The (Euler) homogeneous vector field

$$
\vec{h}=\sum_{i=1}^{n} y_{i} \frac{\partial}{\partial y_{i}}
$$

is polynomial vertical, indeed linear vertical. It is a symmetry of any system of linear differential equations. Hence, it is a global section of $\mathbf{s y m}_{X}$.

The definitions of degree and homogeneous components of a polynomial vertical vector field are clear. Given a function field $\mathcal{K}$ of meromorphic functions on $\mathcal{V} \subseteq \mathcal{U}$, we can also speak of the polynomial vertical vector fields with coefficients in $\mathcal{K}$. They are the polynomial vertical vector fields

$$
Y=\sum_{i=1}^{n} P_{i}(x, y) \frac{\partial}{\partial y_{i}},
$$

where $P_{1}(x, y), \ldots, P_{n}(x, y)$ are in $\mathcal{K}\left[y_{1}, \ldots, y_{n}\right]$.

\subsection{Homogeneous components of symmetries}

Let us consider a vertical infinitesimal symmetry $Y \in \operatorname{sym}_{X}(\mathcal{V})$ with $\mathcal{V} \subseteq \mathcal{U}$. We can develop the Maclaurin series for the components of $Y$ with respect the variables $y_{1}, \ldots, y_{n}$, obtaining

$$
Y=\sum_{i=1}^{n} \sum_{\alpha \in \mathbb{Z}_{+}^{n}} g_{i, \alpha}(x) y^{\alpha} \frac{\partial}{\partial y_{i}},
$$

where the functions $g_{i, \alpha}(x)$ are analytic functions on $\mathcal{V}$. We decompose $Y$ as a sum of its homogeneous components

$$
Y=Y_{0}+Y_{1}+Y_{2}+\cdots
$$

where each $Y_{j}$ is a homogeneous polynomial vertical vector field of degree $j$ (i.e., with respect to the $y$ variables) in $\mathcal{V} \times \mathbb{C}^{n}$.

Lemma 3.3. Let $Y \in \mathbf{s y m}_{X}(\mathcal{V})$ with $\mathcal{V} \subseteq \mathcal{U}$ be a symmetry of $X$. All the homogeneous components $Y_{j}$ of its Maclaurin series are symmetries of $X$.

Proof. The Lie bracket can be computed componentwise because the map $[X, \bullet]$ is homogeneous of degree 0 in its action on vector fields, so we have

$$
0=[Y, X]=\left[Y_{0}, X\right]+\left[Y_{1}, X\right]+\left[Y_{2}, X\right]+\cdots .
$$

For each $j \geq 0,\left[X, Y_{j}\right]$ is a homogeneous polynomial vertical vector field of degree $j$. Thus, all the terms of the above series vanish and we have proved the result. 
Remark 3.4. The Maclaurin series of $Y$ depends only on the value of $Y$ in small neighbourhood of $\mathcal{V} \times\{0\}$ in $\mathcal{V} \times \mathbb{C}^{n}$. Assume that $Y$ is a rational vertical symmetry of $X$,

$$
Y=\sum_{j=1}^{n} f_{i}(x, y) \frac{\partial}{\partial y_{i}}, \quad f_{i}(x, y) \in \mathbb{C}\left(x, y_{1}, \ldots, y_{n}\right),
$$

whose polar set does not contain the curve $\mathbb{C P}_{1} \times\{0\}$ in $\mathbb{C P}_{1} \times \mathbb{C}^{n}$ (i.e., the denominators do not vanish indentically for $y=\overrightarrow{0}$ ). Then it admits a Maclaurin expansion in $y$ and Lemma 3.3 shows that each homogeneous component $Y_{0}, Y_{1}, Y_{2}$, etc. of $Y$ is a (homogeneous) polynomial vertical symmetry with coefficients in $\mathbb{C}(x)$.

\subsection{Homogeneous polynomial vertical symmetries}

The sheaf $\mathbf{s y m}_{X}$ contains the subsheaf of polynomial vertical symmetries that we denote by $\mathbf{s y m}_{X}^{<\infty}$. Lemma 3.3 implies that the homogeneous components of polynomial vertical symmetries are also symmetries. Hence, we have a decomposition

$$
\mathbf{s y m}_{X}^{<\infty}=\bigoplus_{n=0}^{\infty} \mathbf{s y m}_{X}^{r}
$$

where $\mathbf{s y m}_{X}^{r}$ stands for the sheaf of homogeneous polynomial vertical symmetries of $X$ of degree $r$. These objects can be interpreted simultaneously in two complementary ways, as sheaves and as differential varieties:

(a) As a sheaf, $\mathbf{s y m}_{X}^{r}$ maps each open subset $\mathcal{V} \subseteq \mathcal{U}$ to the set $\operatorname{sym}_{X}^{r}(\mathcal{V})$ of homogeneous polynomial vertical symmetries of $X$ defined in $\mathcal{V} \times \mathbb{C}^{n}$.

(b) As a differential variety, $\mathbf{s y m}_{X}^{r}$ maps each differential field extension $\mathcal{K} \subseteq \mathcal{F}$ to the set $\mathbf{s y m}_{X}^{r}(\mathcal{F})$ of homogeneous polynomial vertical symmetries of $X$ with coefficients in $\mathcal{F}$. Since the Lie bracket is computed algebraically, this makes perfect sense even if $\mathcal{F}$ is not a function field. Our forthcoming Theorem 6.1, stated and proved in Section 6 below, tells that $\mathbf{s y m}_{X}^{r}$ is in fact a linear differential variety defined over $\mathcal{K}$.

If the function field $\mathcal{K}$ contains the rational functions, then rational symmetries, as vector fields in $\mathbb{C P}_{1} \times \mathbb{C}^{n}$, can be always reduced to polynomial vertical symmetries with coefficients in $\mathcal{K}$ :

Proposition 3.5. Assume that $\mathcal{K}$ contains the field of rational functions $\mathbb{C}(x)$. Let $Y$ be a rational vector field in $\mathbb{C P}_{1} \times \mathbb{C}^{n}$ which is a non-characteristic rational symmetry of $X$ and whose polar subset does not include the curve $\mathcal{U} \times\{0\}$ in $\mathcal{U} \times \mathbb{C}^{n}$. We consider the Maclaurin series

$$
Y-(Y x) X=Y_{0}+Y_{1}+Y_{2}+\cdots,
$$

where each $Y_{r}$ is a homogeneous polynomial vertical vector field of degree $r$. Then, for each $r$, $Y_{r} \in \mathbf{s y m}_{X}^{r}(\mathcal{K})$ and for at least one index $r, Y_{r}$ is not zero.

Proof. Lemma 3.3 and its Remark 3.4 show that the vector fields $Y_{r}$ are symmetries. We only need to check that they have coefficients in $\mathcal{K}$. Let us consider the expression of $Y$ in coordinates

$$
Y=h(x, y) \frac{\partial}{\partial x}+\sum_{j=1}^{n} f_{j}(x, y) \frac{\partial}{\partial y_{j}} .
$$


Then

$$
Y-(Y x) X=\sum_{j=1}^{n} H_{j}(x, y) \frac{\partial}{\partial y_{j}}, \quad H_{j}(x, y)=\sum_{i=1}^{n}\left(f_{j}(x, y)-h(x, y) a_{j i}(x) y_{i}\right),
$$

and

$$
Y_{r}=\sum_{j=1}^{n} \sum_{|\alpha|=r} \frac{\partial^{|\alpha|} H_{j}}{\partial y^{\alpha}}(x, 0) \frac{y^{\alpha}}{\alpha !} \frac{\partial}{\partial y_{j}} .
$$

A direct examination of the expressions shows that they have coefficients in $\mathcal{K}$.

Since the Lie bracket is a graded operation, the sheaves $\mathbf{s y m}_{X}^{r}$ are not in general Lie algebra sheaves. We have

$$
[,]: \mathbf{s y m}_{X}^{r} \times \mathbf{s y m}_{X}^{s} \rightarrow \mathbf{s y m}_{X}^{r+s-1} .
$$

For $n>1$ only $\mathbf{s y m}_{X}^{0}, \mathbf{s y m}_{X}^{1}$ and $\mathbf{s y m}_{X}^{0} \oplus \mathbf{s y m}_{X}^{1}$ are Lie algebra sheaves. Our next objective is to show that the sections of $\mathbf{s y m}_{X}^{r}$ for each $r$ are solutions of a hierarchy of linear differential systems canonically attached to (2.1).

\section{Polynomial vector fields in $\mathbb{C}^{n}$}

\subsection{The Lie algebra of polynomial vector fields}

Polynomial vertical symmetries are polynomial vector fields along the fibers of the projection $\mathcal{U} \times \mathbb{C}^{n} \rightarrow \mathcal{U}$. In this section we will give some remarks about the structure of the Lie algebra $\mathfrak{X}\left[\mathbb{C}^{n}\right]^{<\infty}$ of polynomial vector fields in $\mathbb{C}^{n}$

$$
\mathfrak{X}\left[\mathbb{C}^{n}\right]^{<\infty}=\left\{\sum_{i=1}^{n} P_{i}(y) \frac{\partial}{\partial y_{i}}: P_{i}(y) \in \mathbb{C}\left[y_{1}, \ldots, y_{n}\right]\right\} .
$$

By taking homogeneous components, we have $\mathfrak{X}\left[\mathbb{C}^{n}\right]=\bigoplus_{r=0}^{\infty} \mathfrak{X}^{r}\left[\mathbb{C}^{n}\right]$, where

$$
\mathfrak{X}^{r}\left[\mathbb{C}^{n}\right]=\left\{\sum_{i=1}^{n} P_{i}(y) \frac{\partial}{\partial y_{i}}: P_{i}(y) \text { homogeneous of degree } r\right\} .
$$

The Lie bracket respects the degree in the following way

$$
[,]: \mathfrak{X}^{r}\left[\mathbb{C}^{n}\right] \times \mathfrak{X}^{s}\left[\mathbb{C}^{n}\right] \rightarrow \mathfrak{X}^{r+s-1}\left[\mathbb{C}^{n}\right]
$$

Remark 4.1. For $n>2$, exactly two of the homogeneous components of $\mathfrak{X}\left[\mathbb{C}^{n}\right]$ are Lie subalgebras:

(a) The homogeneous component of degree zero $\mathfrak{X}^{0}\left[\mathbb{C}^{n}\right]$. It is the Lie algebra of the infinitesimal generators of the action of the group of translations in $\mathbb{C}^{n}$. It is an abelian Lie algebra canonically isomorphic to $\mathbb{C}^{n}$

$$
\mathfrak{X}^{0}\left[\mathbb{C}^{n}\right] \simeq \mathbb{C}^{n}, \quad \frac{\partial}{\partial y_{j}} \mapsto e_{j},
$$

where $\left\{e_{1}, \ldots, e_{n}\right\}$ stands for the canonical basis of $\mathbb{C}^{n}$. 
(b) The homogeneous component of degree one, $\mathfrak{X}^{1}\left[\mathbb{C}^{n}\right]$. It is the Lie algebra of linear vector fields in $\mathbb{C}^{n}$. It consists of the infinitesimal generators of the action of the group of linear transformations of $\mathbb{C}^{n}$. It is canonically isomorphic to $\mathfrak{g l}(n, \mathbb{C})$ in the following sense:

$$
\mathfrak{X}^{1}\left[\mathbb{C}^{n}\right] \simeq \mathfrak{g l}(n, \mathbb{C}), \quad \sum_{i, j=1}^{n} a_{i j} y_{j} \frac{\partial}{\partial y_{i}} \mapsto A,
$$

where $A$ stands for $n \times n$ matrix of entries $a_{i j}$.

Given an endomorphism $A \in \mathfrak{g l}(n, \mathbb{C})$, we let $\vec{v}_{A}$ denote its corresponding linear vector field in $\mathbb{C}^{n}$

$$
\vec{v}_{A}:=\sum_{i, j=1}^{n} a_{i j} y_{j} \frac{\partial}{\partial y_{i}} \quad \text { with the identity } \quad \vec{v}_{A}(y)=\left.\frac{d}{d \varepsilon}\right|_{\varepsilon=0} e^{\varepsilon A} y .
$$

We may easily check that this morphism is in fact an anti-isomorphism of Lie algebras: for any pair $(A, B)$ of matrices, we have

$$
\left[\vec{v}_{A}, \vec{v}_{B}\right]+\vec{v}_{[A, B]}=0 .
$$

\subsection{Induced linear actions}

Let us consider the canonical action of $\operatorname{GL}(n, \mathbb{C})$ on $\mathbb{C}^{n}$ by linear transformations

$$
\mathrm{GL}(n, \mathbb{C}) \times \mathbb{C}^{n} \rightarrow \mathbb{C}^{n}, \quad(A, y) \mapsto A y,
$$

By abuse of notation, we denote non degenerate matrices and their associated linear transformation on $\mathbb{C}^{n}$ by the same symbols. Let $A$ be a linear transformation and $Y$ be a homogeneous polynomial vector field of degree $r$.

Viewing $A$ as a (linear) diffeomorphism of $\mathbb{C}^{n}$, we let $A_{*}(Y)$ denote the vector field transformed by $A$. In general, for any diffeomorphism $F$, we let $F_{*}(Y)(F(p))=d F(Y(p))$, i.e.,

$$
F_{*}(Y)(p)=d F\left(Y\left(F^{-1}(p)\right)\right) .
$$

This defines a natural action of diffeomorphisms of $\mathbb{C}^{n}$ on vector fields of $\mathbb{C}^{n}$.

It is easy to check that $A_{*}(Y)$ is also a homogeneous polynomial vector field of degree $r$. Thus, for each $r \geq 0$ we have an induced representation

$$
\Phi_{r}: \mathrm{GL}(n, \mathbb{C}) \rightarrow \mathrm{GL}\left(\mathfrak{X}^{r}\left[\mathbb{C}^{n}\right]\right), \quad \Phi_{r}(A)(Y)=A_{*}(Y),
$$

which yields a linear representations of $\operatorname{GL}(n, \mathbb{C})$ on the finite-dimensional vector spaces $\mathfrak{X}^{r}\left[\mathbb{C}^{n}\right]$. This action can be differentiated at the identity obtaining the infinitesimal action

$$
\Phi_{r}^{\prime}: \mathfrak{g l}(n, \mathbb{C}) \rightarrow \operatorname{End}\left(\mathfrak{X}^{r}\left[\mathbb{C}^{n}\right]\right), \quad \Phi_{r}^{\prime}(A)(Y)=\left.\frac{d}{d \varepsilon}\right|_{\varepsilon=0} \Phi\left(e^{\varepsilon A}\right)(Y) .
$$

The following remark is key to connect the definition of symmetry with the differential Galois theoretic aspects of equation (2.1).

Lemma 4.2. The infinitesimal action $\Phi^{\prime}$ of $\mathfrak{g l}(n, \mathbb{C})$ in $\mathfrak{X}^{r}\left[\mathbb{C}^{n}\right]$ coincides up to a change of sign, by the canonical isomorphism between $\mathfrak{X}^{1}\left[\mathbb{C}^{n}\right]$ and $\mathfrak{g l}(n, \mathbb{C})$, with the Lie bracket action of linear vector fields on $\mathfrak{X}^{r}\left[\mathbb{C}^{n}\right]$. That is, for any endomorphism $A$ and homogeneous polynomial vector field $Y$ in $\mathbb{C}^{n}$

$$
\Phi_{r}^{\prime}(A)(Y)=-\left[\vec{v}_{A}, Y\right] .
$$

Proof. Let us define, for each $A$ and $\varepsilon, \sigma_{\varepsilon}: \mathbb{C}^{n} \rightarrow \mathbb{C}^{n}$, the map that sends each $y \in \mathbb{C}^{n}$ to $e^{\varepsilon A} y$. Thus, $\left\{\sigma_{\varepsilon}\right\}_{\varepsilon \in \mathbb{C}}$ is the flow of the vector field $\vec{v}_{A}$. We have

$$
\Phi_{r}^{\prime}(A)(Y)=\left.\frac{d}{d \varepsilon}\right|_{\varepsilon=0} \Phi\left(e^{\varepsilon A}\right)(Y)=\left.\frac{d}{d \varepsilon}\right|_{\varepsilon=0} \sigma_{\varepsilon *}(Y)=-\operatorname{Lie}_{\vec{v}_{A}} Y=-\left[\vec{v}_{A}, Y\right],
$$

by the usual geometric definition of Lie derivative. 


\section{$5 \quad$ Lie-Vessiot hierarchy and Galois group}

\subsection{The Lie-Vessiot hierarchy}

Here we recall some of the definitions from [5], adapted to the particular case of linear equations. Let $E$ be a finite-dimensional complex vector space and $(E, \Psi)$ a linear representation of $\operatorname{GL}(n, \mathbb{C})$ in $E$. The group morphism $\Psi$ induces a Lie algebra morphism $\Psi^{\prime}$ :

$$
\Psi: \operatorname{GL}(n, \mathbb{C}) \mapsto \mathrm{GL}(E), \quad \Psi^{\prime}: \mathfrak{g l}(n, \mathbb{C}) \rightarrow \operatorname{End}(E) .
$$

This morphism transports our linear differential system (2.1) to a linear differential system in $\mathcal{U} \times E$ with coefficients in $\mathcal{K}$ :

$$
\frac{d v}{d x}=\Psi^{\prime}(A(x))(v) .
$$

We say that system (5.1) is the Lie-Vessiot system induced by (2.1) in the representation $(E, \Psi)$. They are the geometric analog of the differential systems obtained by Tannakian correspondence on tensor constructions in standard differential Galois theory, see [10, 21].

A solution of the Lie-Vessiot system (5.1) in $\mathcal{V} \subset \mathcal{U}$ is an analytic map $\mathcal{V} \rightarrow E$ that satisfies the equations. Given a differential field extension $\mathcal{K} \subseteq \mathcal{F}$, a solution of the Lie-Vessiot system (5.1) in $\mathcal{F}$ can be thought of as being an element of $E \otimes_{\mathbb{C}} \mathcal{F}$. If we take a basis $v_{1}, \ldots, v_{n}$ of $E$ and denote by $\lambda_{1}, \ldots, \lambda_{n}$ their corresponding linear coordinate functions, the functions $b_{i j}(x)=\lambda_{i}\left(\Psi^{\prime}(A(x)) v_{j}\right)$ are elements of $\mathcal{K}$ and the differential equation can be written in coordinates

$$
\frac{d \lambda}{d x}=B(x) \lambda, \quad \lambda=\left(\lambda_{1}, \ldots, \lambda_{m}\right), \quad B(x) \in \mathfrak{g l}(m, \mathcal{K}) .
$$

Note that, as the Lie-Vessiot construction is a Lie algebra morphism, the poles of $B(x)$ are exactly the poles of $A(x)$.

There is a natural relation between solutions of (2.1) and of its induced Lie-Vessiot system (5.1): if $M(x)$ is a fundamental matrix of solutions of (2.1) defined in $\mathcal{V} \subseteq \mathcal{U}$ then, for all $v_{0} \in E, v(x)=\Psi(M(x)) v_{0}$ is a particular solution of the Lie-Vessiot system (5.1).

\subsection{The Galois group}

The Lie-Vessiot systems induced by (2.1) form a hierarchy of differential equations which encode the differential algebraic properties of (2.1). It allows us to give a "geometric" definition of the differential Galois group. In this definition we are concerned with two kind of solutions of the Lie-Vessiot systems:

(a) We say that a solution $v(x)$ of the Lie-Vessiot system (5.1) is $\mathcal{K}$-rational if it belongs to $E \otimes_{\mathbb{C}} \mathcal{K}$. This means that $v(x)$ has its coordinates $(5.2)$ in $\mathcal{K}$.

(b) We say that an element $w(x)$ of $E \otimes_{\mathbb{C}} \mathcal{K}$ is a $\mathcal{K}$-exponential pre-solution of the Lie-Vessiot system (5.1) if there is a function $b(x) \in \mathcal{K}$ such that

$$
\frac{d w}{d x}-\Psi^{\prime}(A(x))(w)=-b(x) w
$$

It models the case in which $v(x)=\exp \left(\int b(x) d x\right) w(x)$ is a solution of the Lie-Vessiot system (5.1) or, equivalently, the class $\langle v(x)\rangle$ is a rational solution of the equation in the projective space $\mathbb{P}(E)$ obtained by reducing the Lie-Vessiot system (5.1) by the Euler homogeneous vector field (see Example 3.2) of symmetries. 
Note that the concept of $\mathcal{K}$-exponential pre-solution extends that of $\mathcal{K}$-rational solution: any $\mathcal{K}$-rational solution is a $\mathcal{K}$-exponential pre-solution in which the multiplier $b(x)$ vanishes.

Definition 5.1. Let us fix an $x_{0} \in \mathcal{U}$ which is not a singularity of (2.1). We say that a nondegenerate matrix $\sigma \in \operatorname{GL}(n, \mathbb{C})$ is Galoisian at $x_{0}$ if for any linear representation $(E, \Psi)$ it satisfies the two following conditions:

(a) For any $\mathcal{K}$-rational solution $v(x)$ of any induced Lie-Vessiot system $(5.1), \Psi(\sigma)\left(v\left(x_{0}\right)\right)=$ $v\left(x_{0}\right)$. Note that, if $v(x)$ is a $\mathcal{K}$-rational solution then $v\left(x_{0}\right)$ is well defined as an element of $E$.

(b) For any $\mathcal{K}$-exponential pre-solution $w(x)$ of any induced Lie-Vessiot system (5.1), for which $w\left(x_{0}\right)$ is well defined, $w\left(x_{0}\right)$ is an eigenvector of $\Psi(\sigma)$.

The Galoisian matrices at $x_{0}$ form a group $\operatorname{Gal}\left(x_{0}, X\right)$, called the Galois group of (2.1) at the point $x_{0}$. It is the stabilizer of all the values at $x_{0}$ of $\mathcal{K}$-rational solutions, and the lines spanned by the values at $x_{0}$ of $\mathcal{K}$-exponential pre-solutions of induced Lie-Vessiot systems.

Although this geometric definition may seem different from the standard ones from PicardVessiot theory, it produces the same group. Choose a normalized local solution matrix at $x_{0}$, i.e., one with initial condition being the identity at the point $x_{0}$; then $v\left(x_{0}\right)$ will be the coordinates of the invariant $v(x)$ on this normalized basis of solutions. Our definition hence says that a mat$\operatorname{rix} \sigma$ is in $\operatorname{Gal}\left(x_{0}, X\right)$ if and only if it admits all the (semi-)invariants of the (Picard-Vessiot) differential Galois group as (semi-)invariants.

The following facts are well known in differential Galois theory (we refer the interested reader to $[8,21]$ for a general exposition, or to $[4,5]$ for an exposition which is consistent with our geometric definition):

(a) The Galois group $\operatorname{Gal}\left(x_{0}, X\right)$ is an algebraic subgroup of $\operatorname{GL}(n, \mathbb{C})$.

(b) The Galois groups at two different non-singular points $x_{0}$ and $x_{1}$ are conjugated. We will write $\operatorname{Gal}(X)$ to denote this abstract Galois group, that we call the Galois group of the equation $(2.1)$ over $\mathcal{K}$.

(c) The system (2.1) is integrable by Liouvillian functions if and only if $\operatorname{Gal}\left(x_{0}, X\right)$ is a virtually solvable group, i.e., its identity component is solvable.

The next lemma encodes the expected Galois correspondence. The reader may check [4, Proposition 5.4] for a geometric proof that relies on Lie's reduction method and Chevalley theorem.

Lemma 5.2. Let $(E, \Psi)$ be a linear representation of $\mathrm{GL}(n, \mathbb{C})$ and let $z(x)$ be a solution of its corresponding induced Lie-Vessiot system (5.1). Then, $z(x)$ is a $\mathcal{K}$-rational solution if and only if for all Galoisian matrices at $x_{0}, \sigma \in \operatorname{Gal}\left(x_{0}, X\right)$, we have $\Psi(\sigma) z\left(x_{0}\right)=z\left(x_{0}\right)$.

\section{Symmetries vs Galois}

\subsection{Symmetries as solutions of the Lie-Vessiot hierarchy}

A key of the relation between the Galois group and the symmetries is the fact that polynomial vertical vector fields in $\mathcal{V} \subseteq \mathcal{U}$ of can be seen as maps $\mathcal{V} \rightarrow \mathfrak{X}\left[\mathbb{C}^{n}\right]$. For a given polynomial vertical vector field $Y$ and $x_{0} \in \mathcal{V}$ we will write $Y\left(x_{0}\right)$ for the value of $Y$ at $x_{0}$. It is a polynomial vector field in $\mathbb{C}^{n}$ that corresponds to the restriction of $Y$ to the fibre $\left\{x_{0}\right\} \times \mathbb{C}^{n}$. It is clear that, for general vertical vector fields, $[Y, Z]\left(x_{0}\right)=\left[Y\left(x_{0}\right), Z\left(x_{0}\right)\right]$. If we restrict our considerations to homogeneous polynomial vertical vector fields of a fixed degree $r$, then $\mathfrak{X}^{r}\left[\mathbb{C}^{n}\right]$ turns out to be a finite-dimensional complex space. This will allow us to describe polynomial vertical symmetries as solutions of some systems of the Lie-Vessiot hierarchy. 
Theorem 6.1. Let $Y$ be a homogeneous polynomial vertical vector field of degree $r$ defined in $\mathcal{V} \times \mathbb{C}^{n}$ with $\mathcal{V} \subseteq \mathcal{U}$. Then, $Y$ is a symmetry of (2.1) if and only if, as a map from $\mathcal{V}$ to $\mathfrak{X}^{r}\left[\mathbb{C}^{n}\right]$, it is a solution of the Lie-Vessiot system induced in the representation $\left(\Phi_{r}, \mathfrak{X}^{r}\left[\mathbb{C}^{n}\right]\right)$ from Section 4.2 .

In other words, $Y \in \mathbf{s y m}_{X}(\mathcal{V})$ if and only if it satisfies

$$
\frac{d Y}{d x}=\Phi_{r}^{\prime}(A(x)) Y \text {. }
$$

Proof. Recall that if $\vec{v}_{A}=\vec{v}_{A}(x)$ is the linear vertical vector field in $\mathcal{U} \times \mathbb{C}^{n}$ corresponding to the matrix $A(x)$, then $X=\frac{\partial}{\partial x}+\vec{v}_{A}(x)$. Let us compute the Lie bracket

$$
[X, Y]=\left[\frac{\partial}{\partial x}+\vec{v}_{A}(x), Y\right]=\frac{d Y}{d x}+\left[\vec{v}_{A}(x), Y\right]
$$

Thus, $Y$ is a symmetry if and only if $\frac{d Y}{d x}=-\left[\vec{v}_{A}(x), Y\right]$. Finally, by Lemma 4.2 we have that $Y$ satisfies the stated differential equation if and only if it is a symmetry.

If we denote by $\mathfrak{X}\left[\mathbb{C}^{n}\right]<\infty$ the polynomial vector fields in $\mathbb{C}^{n}$, then:

Corollary 6.2. Let $x_{0}$ be a non-singular point, and $\mathcal{V}$ a simply-connected neighbourhood of $x_{0}$ in $\mathcal{U}$. Then, for each polynomial vector field $Y^{(0)} \in \mathfrak{X}\left[\mathbb{C}^{n}\right]^{<\infty}$ there is a unique polynomial vertical symmetry $Y \in \mathbf{s y m}_{X}(\mathcal{V})^{<\infty}$ such that $Y\left(x_{0}\right)=Y^{(0)}$. Moreover, $\mathbf{s y m}_{X}(\mathcal{V})^{<\infty}$ and $\mathfrak{X}\left[\mathbb{C}^{n}\right]<\infty$ are isomorphic Lie algebras.

Proof. The map $\operatorname{sym}_{X}(\mathcal{V})^{<\infty} \rightarrow \mathfrak{X}\left[\mathbb{C}^{n}\right]^{<\infty}, Y \mapsto Y\left(x_{0}\right)$, is a Lie algebra morphism since the computation of the Lie bracket and the restriction to the fiber $\left\{x_{0}\right\} \times \mathbb{C}^{n}$ are commuting processes. We have to see that it is an isomorphism. Let $r$ be the degree of $Y^{(0)}$ and

$$
Y^{(0)}=Y_{0}^{(0)}+Y_{1}^{(0)}+\cdots+Y_{r}^{(0)}
$$

the decomposition of $Y^{(0)}$ in homogeneous components. Let $Y_{k}$ be the solution in $\mathcal{V}$ of the Cauchy problem

$$
\frac{d Y_{k}}{d x}=\Phi_{j}^{\prime}(A(x)) Y_{k}, \quad Y_{k}\left(x_{0}\right)=Y_{k}^{(0)} .
$$

The existence and uniqueness of the solution guarantees that

$$
Y=Y_{0}+Y_{1}+\cdots+Y_{r}
$$

is the only polynomial vertical symmetry such that $Y\left(x_{0}\right)=Y^{(0)}$.

We will now write the system (6.1) in more explicit form using tensor products.

Proposition 6.3. Let $N:=\left(\begin{array}{c}n+m-1 \\ m\end{array}\right)$ denote the number of monomials of degree $m$ in $n$ variables. We define the matrix $\mathcal{A}_{m}:=A \otimes \operatorname{Id}_{N}+\operatorname{Id}_{n} \otimes \mathbf{s y m}^{m}\left(A^{\star}\right)$ of size $n N$.

The system $y^{\prime}=\mathcal{A}_{m} y$ has a rational solution

$$
\begin{aligned}
y= & {\left[a_{1,1}(x), \ldots, a_{N, 1}(x), \ldots, a_{1, j}(x), \ldots, a_{N, j}(x), \ldots,\right.} \\
& \left.a_{1, n}(x), \ldots, a_{N, n}(x)\right]^{T}, \quad a_{i, j}(x) \in \mathcal{K}
\end{aligned}
$$

if and only if $X$ admits the homogeneous vertical symmetry

$$
Y=\sum_{j=1}^{n}\left(\sum_{i=1}^{N} a_{i, j}(x) \mu_{i}\left(y_{1}, \ldots, y_{n}\right)\right) \frac{\partial}{\partial y_{j}},
$$

where $\mu_{i}\left(y_{1}, \ldots, y_{n}\right)$ denotes the $i$-th monomial (for the lexicographic order) of degree $m$ in the $n$ variables $y_{1}, \ldots, y_{n}$. 
Proof. Let $(\mathcal{M}, \partial)$ denote the differential module associated with $y^{\prime}=A y$. Letting $y_{1}, \ldots, y_{n}$ denote a basis of the dual $\mathcal{M}^{\star}$, we see that $X$ represents the action of $\partial$ on $\mathcal{M}^{\star}(X$ acts on the first integrals rather than on the solutions, see $[13,15,22])$. The monomials of degree $m$ in the $y_{1}, \ldots, y_{n}$ form a basis of $\operatorname{Sym}^{m}\left(\mathcal{M}^{\star}\right)$. So, a homogeneous vertical polynomial symmetry is a map from $\mathcal{M}^{\star}$ to $\operatorname{Sym}^{m}\left(\mathcal{M}^{\star}\right)$ which furthermore commutes with $X$ (and hence with $\partial$ ). Now

$$
\operatorname{Hom}\left(\mathcal{M}^{\star}, \operatorname{Sym}^{m}\left(\mathcal{M}^{\star}\right)\right)=\left(\mathcal{M}^{\star}\right)^{\star} \otimes \operatorname{Sym}^{m}\left(\mathcal{M}^{\star}\right)=\mathcal{M} \otimes \operatorname{Sym}^{m}\left(\mathcal{M}^{\star}\right),
$$

so that

$$
\operatorname{Hom}_{\partial}\left(\mathcal{M}^{\star}, \operatorname{Sym}^{m}\left(\mathcal{M}^{\star}\right)\right)=\operatorname{ker}\left(\partial, \mathcal{M} \otimes \operatorname{Sym}^{m}\left(\mathcal{M}^{\star}\right)\right) .
$$

This shows that the coefficients of $Y$ are exactly the rational solutions of $y^{\prime}=\mathcal{A}_{m} y$.

\subsection{Symmetries vs Galois}

The intrinsic relation between the Galois group and the Lie algebra of symmetries of (5.2) is made explicit by the following result.

Theorem 6.4. Let $Y^{(0)}$ be a polynomial vector field in $\mathbb{C}^{n}$, and $x_{0}$ a non-singular point of (2.1). There is a polynomial vertical symmetry $Y$ of $X$ with coefficients in $\mathcal{K}$ such that $Y\left(x_{0}\right)=Y^{(0)}$ if and only if for each Galoisian matrix $\sigma \in \operatorname{Gal}\left(x_{0}, X\right), \sigma_{*} Y^{(0)}=Y^{(0)}$.

Proof. It follows from our definition of Galois group. By Theorem 6.2, for each one of them there is a polynomial vertical $Y$ symmetry such that $Y\left(x_{0}\right)=Y^{(0)}$ defined in a neigbourhood of $x_{0}$. By Theorem 6.1 this is a solution of a Lie-Vessiot system induced by $X$, here we consider all the homogeneous components simultaneously. Finally, by Lemma 5.2, this solution has coefficients in $\mathcal{K}$ if and only if $A_{*} Y\left(x_{0}\right)=Y\left(x_{0}\right)$ for all Galoisian matrices at $x_{0}$.

Hence, the Galois group $\operatorname{Gal}\left(x_{0}, X\right)$ of $(2.1)$ determines the Lie algebra $\mathbf{s y m}^{<\infty}(\mathcal{K})$ of polynomial vertical $\mathcal{K}$-rational symmetries in the following sense. The Lie algebra $\mathbf{s y m}^{<\infty}(\mathcal{K})$ is isomorphic to $\mathfrak{X}\left[\mathbb{C}^{n}\right]^{\operatorname{Gal}\left(x_{0}, X\right)}$, the Lie algebra of polynomial vector fields fixed by the action of $\operatorname{Gal}\left(x_{0}, X\right)$ by linear transformations in $\mathbb{C}^{n}$. However, we do not have a reciprocal: in general, the Galois group is contained in the stabilizer of the Lie algebra of $\mathcal{K}$-rational symmetries.

It is possible also to dualize the situation and to consider the Galois group itself as symmetries of the infinitesimal symmetries of equation (2.1) as follows. Given a polynomial vector field $Y$ in $\mathbb{C}^{n}$ a linear symmetry of $Y$ is a non-degenerated matrix $\sigma$ such that $\sigma_{*} Y=Y$. Here, $\sigma$ stands for the transformation

$$
y=\left(y_{1}, \ldots, y_{n}\right) \rightarrow \sigma y=\left(\sum_{j=1}^{n} \sigma_{1 j} y_{j}, \ldots, \sum_{j=1}^{n} \sigma_{n j} y_{j}\right) .
$$

If the expression in coordinates of $Y$ is

$$
Y=\sum P_{i}(y) \frac{\partial}{\partial y_{i}}
$$

then the matrix $\sigma$ is linear symmetry of $Y$ if and only if it satisfies the equations

$$
P_{i}(\sigma y)=\sum_{j=1}^{n} \sigma_{i j} P_{j}(y) .
$$

If we are looking for the linear symmetries of a homogeneous polynomial vector field of degree $m$, it yields a total of $n \times\left(\begin{array}{c}n+m-1 \\ m\end{array}\right)$ equations. Thus, for generic polynomial vector fields of high degree the group of linear symmetries reduces to the identity. 
Example 6.5. Let us compute the symmetries of the quadratic vector field

$$
Y=y_{2}^{2} \frac{\partial}{\partial y_{1}}
$$

in $\mathbb{C}^{2}$. Equations (6.2) for this particular case yield

$$
\sigma_{21}^{2} y_{1}^{2}+2 \sigma_{21} \sigma_{22} y_{1} y_{2}+\sigma_{22}^{2} y_{2}^{2}=\sigma_{11} y_{2}^{2}, \quad 0=\sigma_{21} y_{2}^{2},
$$

equating each coefficient, we obtain

$$
\sigma_{11}=\sigma_{22}^{2}, \quad \sigma_{21}=0 .
$$

Thus, the group of linear symmetries is

$$
\left\{\left(\begin{array}{cc}
\lambda^{2} & \mu \\
0 & \lambda
\end{array}\right): \lambda \in \mathbb{C}^{*}, \mu \in \mathbb{C}\right\} .
$$

The Lie-Vessiot induced system for polynomial vertical symmetries of arbitrary degree $r$ is

$$
\frac{d Y}{d x}=-\left[\vec{v}_{A}(x), Y\right]
$$

We can consider all the homogeneous components simultaneously. Theorem 6.4 can be restated in the following terms:

Corollary 6.6. Let $Y^{(0)}$ be a polynomial vector field in $\mathbb{C}^{n}$, and $x_{0}$ a non-singular point of equation (2.1). The necessary and sufficient condition for the existence of a polynomial vertical $\mathcal{K}$-rational symmetry $Y$ of $X$ such that $Y\left(x_{0}\right)=Y^{(0)}$ is that the Galois group $\operatorname{Gal}\left(x_{0}, X\right)$ is contained in the group of linear symmetries of $Y_{0}$.

Example 6.7. Let us consider the system

$$
\frac{d y_{1}}{d x}=2 a(x) y_{1}+b(x) y_{2}, \quad \frac{d y_{2}}{d x}=a(x) y_{2},
$$

where $a(x), b(x)$, are arbitrary functions in $\mathcal{K}$. A direct computation of the Lie bracket says that $Y=y_{1}^{2} \frac{\partial}{\partial y_{2}}$ is a symmetry, and thus the Galois group of the equation (for any function field $\mathcal{K}$ ) is contained in the group (6.3).

Let us check now how the polynomial vertical symmetries of degrees zero and one look like, and what kind information about the Galois group they carry.

\subsubsection{Symmetries of degree zero}

The canonical isomorphism $\mathfrak{X}^{0}\left[\mathbb{C}^{n}\right] \simeq \mathbb{C}^{n}$ stated in Section 4.1 tell us that the linear representation $\left(\Phi_{0}, \mathfrak{X}^{0}\left[\mathbb{C}^{n}\right]\right)$ is just an isomorphism. In particular, if

$$
Y=\sum_{i=1}^{n} f_{i}(x) \frac{\partial}{\partial y_{i}}
$$

then

$$
\Phi^{\prime}(A(x)) Y=\sum_{i, j=1}^{n} a_{i j}(x) f_{j}(x) \frac{\partial}{\partial y_{i}},
$$

and thus we have the following result: 
Proposition 6.8. A n-tuple of functions $y=\left(\phi_{1}(x), \ldots, \phi_{n}(x)\right)$ is a solution of (2.1) if and only if $Y=\sum_{i=1}^{n} \phi_{i}(x) \frac{\partial}{\partial y_{i}}$ is a symmetry of $(2.1)$.

This proposition can be understood as an infinitesimal version of the superposition principle. If $y(x)$ and $\phi(x)$ are solutions of (2.1), then for all $\varepsilon, y(x)+\varepsilon \phi(x)$ is also a solution. For a fixed $\phi(x)$ and a general $y(x)$, the derivative of this monoparametric family of solutions with respect to $\epsilon$ is a vertical vector field, namely, the symmetry $Y$.

Remark 6.9. Given a symmetry of degree zero of (2.1) (i.e., a solution), we can reduce the system of $n$ differential equations (2.1) to a system of $n-1$ differential equations, by means of a suitable gauge transformation. This simple observation can be viewed as a generalization of the classical result of d'Alembert: the order of a linear differential equation can be reduced by one when a particular solution is known.

\subsubsection{Linear symmetries}

Homogeneous polynomial vertical symmetries of degree one are called linear symmetries. The homogeneous vector field $\vec{h}=\sum_{i=1}^{n} y_{i} \frac{\partial}{\partial y_{i}}$ and its multiples gives us a trivial monoparametric family linear vertical symmetries for any system of differential equations. The canonical isomorphism $\mathfrak{X}^{1}\left[\mathbb{C}^{n}\right] \simeq \mathfrak{g l}(n, \mathbb{C})$ stated in Section 4.1 tell us the that Lie-Vessiot system induced by the representation $\left(\Phi_{1}, \mathfrak{X}^{1}\left[\mathbb{C}^{n}\right]\right)$ can be seen as a matrix equation. If we write

$$
Y=\sum_{i, j=1}^{n} b_{i j} y_{j} \frac{\partial}{\partial y_{i}},
$$

where $B=\left(b_{i j}\right)$ stands for a $n \times n$ matrix of undetermined functions, the induced system is written as

$$
\frac{d B}{d x}=[A(x), B]
$$

This is the equation of isospectral deformations induced by $A(x)$ and has been exhaustively studied. The set of rational solutions of (6.4) is called the eigenring, see [3] for an extensive study of its properties, notably to decompose linear differential systems. If $B(x)$ is a solution of (6.4) it is well known that the Jordan canonical form of $B(x)$ does not depend on the point $x$. Thus, given a linear symmetry $Y$ with matrix $B(x)$, we will classify it according to its Jordan canonical form:

(a) If $B(x)$ has at least two different eigenvalues we will say that $Y$ is a decomposer symmetry.

(b) If all the eigenvalues of $B(x)$ are different we will say that $Y$ is a complete decomposer symmetry.

(b) If the eigenspaces of $B(x)$ are one-dimensional we say that $Y$ is a solver symmetry. That means that its Jordan canonical form does not contain any block of the form

$$
\left(\begin{array}{ll}
\lambda & 0 \\
0 & \lambda
\end{array}\right)
$$

The following theorem is very close to some of the results of C. Jensen in [9, Section 9], on integration by quadratures, and results of M.A. Barkatou in [3] on decomposition - although we obtain it by different means and relate it with the linear symetries and the Galois group of the system. 
Theorem 6.10. The following are equivalent:

(a) There is a decomposer symmetry $Y \in \mathbf{s y m}(\mathcal{K})$ with $k$ different eigenvalues of multiplicity $r_{1}, \ldots, r_{k}$.

(b) The Galois group $\operatorname{Gal}\left(x_{0}, X\right)$ is conjugated to a sugbroup of the group of block-diagonal matrices $\operatorname{GL}\left(r_{1}, \mathbb{C}\right) \times \cdots \times \operatorname{GL}\left(r_{k}, \mathbb{C}\right)$.

Proof. (a) $\Longrightarrow(\mathrm{b})$. Let $x_{0}$ be a non-singular point and $B$ the matrix defined by $Y\left(x_{0}\right)$, with eigenvalues $\lambda_{1}, \ldots, \lambda_{k}$ of multiplicities $r_{1}, \ldots, r_{k}$. Let us consider the decomposition

$$
\mathbb{C}^{n}=E_{1} \oplus \cdots \oplus E_{k}
$$

where the spaces $E_{i}=\operatorname{ker}\left(B-\lambda_{i} \mathrm{Id}\right)$ are the generalized eigenspaces of $B$. The group

$$
G=\left\{\sigma \in \operatorname{GL}(n, \mathbb{C}): \sigma\left(E_{i}\right)=E_{i} \text { for all } i=1, \ldots, k\right\}
$$

is clearly conjugated to the group of block-diagonal matrices. Let us see that all Galoisian matrices at $x_{0}$ are in $G$. If $\sigma$ is Galoisian then, $\sigma_{*}\left(Y\left(x_{0}\right)\right)=Y\left(x_{0}\right)$, but that means $\sigma B \sigma^{-1}=B$, so $\sigma$ conjugates $B$ with itself, and thus it sends generalized eigenspaces of $B$ to themselves.

(b) $\Longrightarrow(\mathrm{a})$. Let us assume that $\operatorname{Gal}\left(x_{0}, X\right)$ is conjugated to a subgroup of the group of blockdiagonal matrices. Then, we have a decomposition of $\mathbb{C}^{n}$ in subspaces as in formula (6.5), such that for all $\sigma \in \operatorname{Gal}\left(x_{0}, X\right)$, and index $i=1, \ldots, k, \sigma\left(E_{i}\right)=E_{i}$. Let us consider the following linear vector fields $\vec{h}_{i}$ in $\mathbb{C}^{n}$ for $i=1, \ldots, k$ defined by properties:

$$
\begin{aligned}
& \left.\vec{h}_{i}\right|_{E_{i}}=\vec{h}, \quad \text { where } \vec{h} \text { stands for the Euler homogeneous vector field, } \\
& \left.\vec{h}_{i}\right|_{E_{j}}=0, \quad \text { if } i \neq j .
\end{aligned}
$$

Let us consider $\mu_{1}, \ldots, \mu_{k}$ different complex numbers and define

$$
Y^{(0)}=\sum_{i=1}^{k} \mu_{i} \vec{h}_{i} .
$$

$Y^{(0)}$ is stabilized by any Galoisian matrix and then, by Lemma 5.2 , there is $\mathcal{K}$-rational symmetry $Y$ whose value at $x_{0}$ is $Y^{(0)}$. This symmetry $Y$ is the decomposer symmetry of the statement.

Corollary 6.11. The following are equivalent:

(a) There is a complete-decomposer linear symmetry in $\mathbf{s y m}_{X}^{1}(\mathcal{K})$.

(b) The Galois group $\operatorname{Gal}\left(x_{0}, X\right)$ is conjugated to subgroup of the group of diagonal matrices $\left(\mathbb{C}^{*}\right)^{n} \subset \mathrm{GL}(n, \mathbb{C})$.

Proof. The statement is the particular case of Theorem 6.10 in which all the eigenvalues are simple.

Remark 6.12. The existence of a complete-decomposer linear symmetry implies the existence of a $n$-dimensional abelian Lie algebra of symmetries. Let us consider a complete-decomposer linear symmetry $Y$ and $x_{0}$ a non-singular point. Let $B$ be the matrix of $Y\left(x_{0}\right)$, and $v_{1}, \ldots, v_{n}$ be a basis of eigenvector of $B$. As before, we define vector fields:

$$
\begin{aligned}
& \vec{h}_{i}\left(v_{i}\right)=\vec{h}\left(v_{i}\right), \quad \text { where } \vec{h} \text { stands for the homogeneous vector field, } \\
& \vec{h}_{i}\left(v_{j}\right)=0, \quad \text { if } i \neq j .
\end{aligned}
$$

It is easy to check that the matrices of the vector fields $\vec{h}_{i}$ have common eigenvectors and then $\left[\vec{h}_{i}, \vec{h}_{j}\right]=0$. For all Galoisian matrix $\sigma$ at $x_{0}$ we have $\sigma_{*}\left(\vec{h}_{i}\right)=\vec{h}_{i}$, and thus by Lemma 5.2 there are linear vertical $\mathcal{K}$-rational symmetries $\vec{H}_{1}, \ldots, \vec{H}_{n}$ such that $\vec{H}_{i}\left(x_{0}\right)=\vec{h}_{i}$. They form a $n$-dimensional abelian Lie algebra. 
Theorem 6.13. If there is a solver symmetry $Y \in \operatorname{sym}(\mathcal{K})$ then the Galois group $\operatorname{Gal}\left(x_{0}, X\right)$ is conjugated to a subgroup of the triangular group.

Proof. Let us consider first the case in which $B$, the matrix of $Y\left(x_{0}\right)$, has only one eigenvalue $\lambda$ of multiplicity $n$. For each Galoisian matrix $\sigma$ at $x_{0}$ we have $\sigma B \sigma^{-1}=B$. We have a chain of subspaces

$$
0 \subset \operatorname{ker}(B-\lambda \mathrm{Id}) \subset \operatorname{ker}(B-\lambda \mathrm{Id})^{2} \subset \cdots \subset \operatorname{ker}(B-\lambda \mathrm{Id})^{n-1} \subset \mathbb{C}^{n} .
$$

In general, $\sigma\left(\operatorname{ker}(B-\lambda \mathrm{Id})^{j}\right)=\operatorname{ker}\left(\sigma B \sigma^{-1}-\lambda \mathrm{Id}\right)^{j}$ and thus Galoisian matrices respect the chain of subspaces. In other words, they are triangular matrices in some suitable basis. For the general case, with different eigenvalues, we first consider the decomposition of the group by block-diagonal matrices given in Theorem 6.10, and then we apply the above argument.

The results of this subsection supported Lie's idea that Lie symmetries are useful for the integrability of the differential equation (2.1) by quadratures or at least its partial integrability or reduction:

(1) By Remark 6.9, the existence of a symmetry of order zero implies a reduction of the order.

(2) The decomposition in block-diagonal form of the Galois group implies that, by means of a gauge transformation, we can transform the equation (2.1) in a direct sum of linear differential equations (Kolchin or Lie-Kolchin reduction, see [5, 21]). By Theorem 6.10, we fall in this case for a decomposer symmetry.

(3) By Remark 6.12 and Theorem 6.13, the existence of either a complete-decomposer or a solver symmetry implies the solvability of the equation by Liouvillian functions.

Also it is not difficult to obtain some results for the Hamiltonian symmetries in the symplectic case, i.e., for non-autonomous linear Hamiltonian systems. In some sense, this approach would shed light on the references $[13,15]$ from the Lie point of view.

\section{A Symmetries of higher-order equations vs first-order systems}

There are two different ways to present the theory of linear differential equations. The first one deals with a single higher-order linear differential equation:

$$
\frac{d^{n} y}{d x^{n}}+a_{n-1}(x) \frac{d^{n-1} y}{d x^{n-1}}+\cdots+a_{0}(x) y=0 .
$$

The second one deals with a system of first-order linear differential equations

$$
\frac{d}{d x}\left(\begin{array}{c}
y_{0} \\
y_{1} \\
\vdots \\
y_{n-1}
\end{array}\right)=\left(\begin{array}{ccccc}
0 & 1 & 0 & \ldots & 0 \\
0 & 0 & 1 & \ldots & 0 \\
\vdots & \vdots & \ddots & \ddots & \vdots \\
-a_{0}(x) & -a_{1}(x) & \ldots & \ldots & -a_{n-1}(x)
\end{array}\right)\left(\begin{array}{c}
y_{0} \\
y_{1} \\
\vdots \\
y_{n-1}
\end{array}\right)
$$

The system (A.2) is called the companion system of (A.1). The variable $y_{i}$ represents the $i$-th derivative of the function $y$. In this paper, we have considered systems instead of higherorder equations. There is a pragmatic reason: although the differential Galois theories for equations (A.1) and (A.2) are the same (see, for instance [21, Section 2.1]), their Lie symmetry theories are not. Higher-order equations have less symmetries than systems of first-order differential equations. 
By definition, an infinitesimal point symmetry of (A.1) is a vector field

$$
Y=\xi(x, y) \frac{\partial}{\partial x}+\eta(x, y) \frac{\partial}{\partial y}
$$

in the plane $x, y$ whose flow maps solutions to solutions. Let us consider the vector field

$$
X=\frac{\partial}{\partial x}+y_{1} \frac{\partial}{\partial y_{0}}+\cdots-\left(a_{0}(x) y_{0}+\cdots+a_{n-1}(x) y_{n-1}\right) \frac{\partial}{\partial y_{n-1}} .
$$

The vector field $Y$ extends to a unique vector field $\tilde{Y}$ in the jet space of coordinates $x, y_{0}$, $\ldots, y_{n-1}$

$$
\tilde{Y}=\xi(x, y) \frac{\partial}{\partial x}+\eta\left(x, y_{0}\right) \frac{\partial}{\partial y_{0}}+\eta_{1}\left(x, y_{0}, y_{1}\right) \frac{\partial}{\partial}+\cdots+\eta_{n-1}\left(x, y_{0}, \ldots, y_{n-1}\right) \frac{\partial}{\partial y_{n-1}},
$$

satisfying the conditions (see [16, Section 2.3])

$$
\begin{aligned}
& {[\tilde{Y}, X] \in(X),} \\
& \operatorname{Lie}_{\tilde{Y}}\left(d y_{0}-y_{1} d x, \ldots, d y_{n-2}-y_{n-1} d x\right) \subseteq\left(d y_{0}-y_{1} d x, \ldots, d y_{n-2}-y_{n-1} d x\right) .
\end{aligned}
$$

It is known that the Lie algebra of point symmetries (in some open subset) of a linear differential equation of order $\geq 2$ is finite-dimensional. The above conditions allow us to generalize the idea of infinitesimal point symmetry. Any vector field in the jet space of coordinates $x, y_{0}, \ldots, y_{n-1}$ is called an infinitesimal contact symmetry if is satisfies

$$
\begin{aligned}
& {[Z, X] \in(X),} \\
& \operatorname{Lie}_{Z}\left(d y_{0}-y_{1} d x, \ldots, d y_{n-2}-y_{n-1} d x\right) \subseteq\left(d y_{0}-y_{1} d x, \ldots, d y_{n-2}-y_{n-1} d x\right) .
\end{aligned}
$$

Infinitesimal point symmetries form a Lie subalgebra of the Lie algebra of infinitesimal contact symmetries.

On the other hand, an infinitesimal symmetry of the system (A.2) is a vector field $Z$ such that $[Z, X] \in(X)$. See for instance [1] and [11, pp. 12-16]. It is clear that the Lie algebra of symmetries of the companion system (A.2) contains the Lie algebra of infinitesimal contact symmetries of the higher-order differential equation (A.1).

In this paper, we have explored the relation between some Lie algebras of symmetries of a firstorder system (A.2) and its differential Galois group. The relation between the Lie algebra of infinitesimal point symmetries of an operator (A.1) and its differential Galois group has been investigated, with rather negative results, in [1] and [17].

\section{Acknowledgements}

The authors thank their colleagues for interesting discussions that encouraged them to write this paper, especially those attending the meeting Algebraic Methods in Dynamical Systems 2014. David Blázquez-Sanz acknowledges Universidad Nacional de Colombia for supporting his research through project ref. HERMES-27984. Juan J. Morales-Ruiz research has been also partially supported by the Spanish MINECO-FEDER Grant MTM2012-31714. We thank the anonymous referees for their remarks and suggestions.

\section{References}

[1] Athorne C., Symmetries of linear ordinary differential equations, J. Phys. A: Math. Gen. 30 (1997), 46394649 . 
[2] Ayoul M., Zung N.T., Galoisian obstructions to non-Hamiltonian integrability, C. R. Math. Acad. Sci. Paris 348 (2010), 1323-1326, arXiv:0901.4586.

[3] Barkatou M.A., Factoring systems of linear functional equations using eigenrings, in Computer Algebra 2006, World Sci. Publ., Hackensack, NJ, 2007, 22-42.

[4] Blázquez-Sanz D., Morales-Ruiz J.J., Differential Galois theory of algebraic Lie-Vessiot systems, in Differential Algebra, Complex Analysis and Orthogonal Polynomials, Contemp. Math., Vol. 509, Amer. Math. Soc., Providence, RI, 2010, 1-58, arXiv:0901.4480.

[5] Blázquez-Sanz D., Morales-Ruiz J.J., Lie's reduction method and differential Galois theory in the complex analytic context, Discrete Contin. Dyn. Syst. 32 (2012), 353-379, arXiv:0901.4479.

[6] Bogoyavlenskij O.I., A concept of integrability of dynamical systems, C. R. Math. Rep. Acad. Sci. Canada 18 (1996), 163-168.

[7] Bogoyavlenskij O.I., Hidden structure of symmetries, Comm. Math. Phys. 254 (2005), 479-488.

[8] Crespo T., Hajto Z., Algebraic groups and differential Galois theory, Graduate Studies in Mathematics, Vol. 122, Amer. Math. Soc., Providence, RI, 2011.

[9] Jensen C.V., Linear ODEs and $\mathcal{D}$-modules, solving and decomposing equations using symmetry methods, Lobachevskii J. Math. 17 (2005), 149-212.

[10] Katz N.M., A conjecture in the arithmetic theory of differential equations, Bull. Soc. Math. France 110 (1982), 203-239.

[11] Kushner A., Lychagin V., Rubtsov V., Contact geometry and nonlinear differential equations, Encyclopedia of Mathematics and its Applications, Vol. 101, Cambridge University Press, Cambridge, 2007.

[12] Malgrange B., On nonlinear differential Galois theory, Chinese Ann. Math. Ser. B 23 (2002), 219-226.

[13] Morales-Ruiz J.J., Differential Galois theory and non-integrability of Hamiltonian systems, Progress in Mathematics, Vol. 179, Birkhäuser Verlag, Basel, 1999.

[14] Morales-Ruiz J.J., Picard-Vessiot theory and integrability, J. Geom. Phys. 87 (2015), 314-343.

[15] Morales-Ruiz J.J., Ramis J.-P., Galoisian obstructions to integrability of Hamiltonian systems, Methods Appl. Anal. 8 (2001), 33-96.

[16] Olver P.J., Applications of Lie groups to differential equations, Graduate Texts in Mathematics, Vol. 107, Springer-Verlag, New York, 1986.

[17] Oudshoorn W.R., van der Put M., Lie symmetries and differential Galois groups of linear equations, Math. Comp. 71 (2002), 349-361.

[18] Seidenberg A., Abstract differential algebra and the analytic case, Proc. Amer. Math. Soc. 9 (1958), $159-164$.

[19] Seidenberg A., Abstract differential algebra and the analytic case. II, Proc. Amer. Math. Soc. 23 (1969), 689-691.

[20] Singer M.F., Testing reducibility of linear differential operators: a group-theoretic perspective, Appl. Algebra Engrg. Comm. Comput. 7 (1996), 77-104.

[21] van der Put M., Singer M.F., Galois theory of linear differential equations, Grundlehren der Mathematischen Wissenschaften, Vol. 328, Springer-Verlag, Berlin, 2003.

[22] Weil J.-A., First integrals and Darboux polynomials of homogeneous linear differential systems, in Applied Algebra, Algebraic Algorithms and Error-Correcting Codes (Paris, 1995), Lecture Notes in Comput. Sci., Vol. 948, Springer, Berlin, 1995, 469-484. 